\title{
Hepatitis B Virus Might Be Sensed by STING-Dependent DNA Sensors and Attenuates the Response of STING-Dependent DNA Sensing Pathway in Humans with Acute and Chronic Hepatitis B Virus Infection
}

\author{
Hongtao Chen, ${ }^{1-3}$ Guirong He, ${ }^{4}$ Yue Chen, ${ }^{4}$ and Xiaoyong Zhang ${ }^{1}$
}

\begin{abstract}
DNA-dependent activator of interferon regulatory factors (DAIs), interferon gamma inducible protein 16 (IFI16), DEAD-box polypeptide 41 (DDX41), DNA-dependent protein kinase (DNA-PK), meiotic recombination 11 homolog A (MRE11), and cyclic GMP-AMP synthase (cGAS) have been identified as intracellular STING-dependent DNA sensors in recent years. Studies have shown that the DNA sensor-STING-interferon (IFN)- $\beta$ pathway plays an important role in the defense against intracellular invasion of many DNA viruses. However, the intracellular recognition of hepatitis B virus (HBV) DNA by DNA sensors is still largely unclear. In this study, we aimed to determine whether the DNA sensor-STING pathway in peripheral blood mononuclear cells (PBMCs) can be activated by acute and chronic HBV infections in humans. We first evaluated the expression of these DNA sensors in PBMCs of acute and chronic HBV-infected patients by quantitative realtime polymerase chain reaction. We next compared the expression of the upregulated DNA sensor between monocytes and nonmonocytes to find its cellular source. Finally, by in vitro stimulation, we analyzed the IFN- $\beta$ response of the DNA sensor-STING pathway in PBMCs and monocytes from chronic HBV-infected patients. The results showed that IFI16, DDX41, MRE11, and the adaptor STING were upregulated in chronic HBVinfected patients, whereas only IFI16 was upregulated in acute HBV-infected patients. However, IFN- $\beta$ expression was not changed in PBMCs from acute and chronic HBV-infected patients. We next found IFI16 was mainly expressed in monocytes of acute and chronic hepatitis B patients. Finally, by stimulation of monocytes with VACV ds 70mer, a ligand for IFI16, we confirmed the attenuated response of the IFI16-STING pathway. Taken together, our results suggest that HBV might be sensed by DNA sensors in PBMCs of acute and chronic HBV-infected patients, and meanwhile HBV infection attenuates the response of the DNA sensor-STING pathway in PBMCs and monocytes, which may facilitate the persistence of HBV infection.
\end{abstract}

Keywords: hepatitis B virus, DNA sensor, innate immunity, hepatitis B

\section{Background}

$\mathbf{H}$ EPATITIS B VIRUS (HBV) is a noncytopathic hepatotropic DNA virus in humans. Infection with $\mathrm{HBV}$ results in acute or chronic hepatitis B (CHB). About 248 million people globally are chronically infected with $\mathrm{HBV}$ and at high risk for liver cirrhosis and hepatocellular carcinoma (1). The mechanism of development of chronicity of HBV infection remains to be determined. The host's age and immune status significantly influence the outcome of acute infection, as the rate of chronicity after acute infection is $>90 \%$ among children $<1$ year and is $<5 \%$ among infected

\footnotetext{
${ }^{1}$ State Key Laboratory of Organ Failure Research, Guangdong Provincial Key Laboratory of Viral Hepatitis Research, Department of Infectious Diseases, Nanfang Hospital, Southern Medical University, Guangzhou, China.

${ }^{2}$ Department of Infectious Diseases, The Second Clinical Medical College (Shenzhen People's Hospital), Jinan University, Shenzhen, China.

${ }^{3}$ Key Laboratory of Pathogenic Microorganisms of Shenzhen, Shenzhen Institute of Respiratory Diseases, Shenzhen, China.

${ }^{4}$ Department of Clinical Laboratory, The Second Clinical Medical College (Shenzhen People's Hospital), Jinan University, Shenzhen, China.

An earlier version of this article was posted as a preprint at Research Square (DOI: 10.21203/rs.2.23304/v1).
} 
adults (21). Thus, the host immune status appears to be a major determinant of the clinical outcome of HBV infection. Besides, HBV is also referred to as a "stealth" virus that elicits negligible innate immune responses in the early infection phase to facilitate the establishment of chronic infection (42). It is well established that a robust and $\mathrm{HBV}$-specific $\mathrm{CD}^{+} \mathrm{T}$ cell response is necessary to eliminate $\mathrm{HBV}$ infection. In contrast, the contribution of the innate immune mechanisms is not well understood and whether HBV can be sensed by the innate immune system remains controversial $(13,23,35)$.

The invasion of mammalian cells by pathogens represents a danger signal for the innate immune system, which takes use of pattern-recognition receptors (PRRs) to recognize conserved motifs from pathogens. The intracellular DNA sensors are responsible for the recognition of cytoplasmic or nuclear pathogen-derived DNA. Recently, DNA-dependent activator of interferon regulatory factors (DAIs), interferon gamma inducible protein 16 (IFI16), DEAD-box polypeptide 41 (DDX41), DNA-dependent protein kinase (DNAPK), meiotic recombination 11 homolog A (MRE11), and cyclic GMP-AMP synthase (cGAS) were identified as intracellular DNA sensors (10). On binding to foreign DNA, these DNA sensors activate stimulator of interferon genes (STING) $(10,27)$, which then activates TANK-binding kinase 1 (TBK1). TBK1 subsequently phosphorylates STING and the downstream transcription factor interferon regulatory factor 3 (IRF3) to promote the expression of type I interferons (IFN-Is) and other cytokines $(3,25,44)$. Thus, the DNA sensor-STING pathway plays a significant role in host defense against invading DNA viruses.

Previous studies have suggested that many viral infectious diseases can induce the activation of STING-dependent DNA-sensing pathways (22), and meanwhile, some viruses utilize a plethora of mechanisms to antagonize the DNAsensing pathways $(30,33,37)$. However, little is known about the roles of DNA sensors in HBV infection. Our previous study showed IFI16 as a component of inflammasome complexes is upregulated in both acute and chronic HBV infections in humans (4). In this study, we scrutinized all the known STING-dependent DNA sensors in peripheral blood mononuclear cells (PBMCs) of acute and chronic HBVinfected patients and found again that only IFI16, expressed most frequently in monocytes, is involved in acute and $\mathrm{CHB}$ in humans. Furthermore, the IFI16-STING pathway is suppressed in $\mathrm{CHB}$, which may contribute to $\mathrm{HBV}$-induced immunotolerance.

\section{Materials and Methods}

\section{Subjects}

From May 2015 to November 2018, 66 treatment-naive patients with chronic HBV infection were recruited at Shenzhen People's Hospital (Shenzhen, China). The patients were classified into three groups according to the guidelines for the treatment of CHB (36): immune-tolerant (IT) carriers $(n=15)$, hepatitis $\mathrm{B}$ e antigen ( $\mathrm{HBeAg})$-positive hepatitis patients (CHB, $n=31)$, and inactive (IA) carriers $(n=20)$. Twenty-eight healthy individuals were enrolled as healthy controls (HCs). Patients with acute hepatitis B (AHB, $n=8)$ and varicella zoster virus (VZV, $n=8$ ) were also included in this study. Patients with any other disease were excluded. The clinical characteristics of HCs and patients are shown in Tables 1 and 2. This study was approved by the Ethical Committee of Shenzhen People's Hospital. All participants signed written informed consent before sample collection.

\section{Isolation of PBMCs and monocytes}

Blood samples were obtained from each subject. PBMCs were isolated using Ficoll-Paque (GE Healthcare) density gradient centrifugation. $\mathrm{CD}_{1}{ }^{+}$monocytes and nonmonocytes were isolated from fresh PBMCs using Dynabeads untouched human monocytes separation kits (Invitrogen, Shanghai, China). FACS analysis showed that the purity of the obtained $\mathrm{CD}_{14}{ }^{+}$monocytes was $>95 \%$.

\section{RNA extraction, reverse transcription, and quantitative real-time polymerase chain reaction}

Total RNA was extracted from cells with TRIzol (Invitrogen). Target mRNA was reverse transcribed using the PrimeScript RT reagent Kit with gDNA Eraser (TaKaRa). The primer sets used in this study are shown in Table 3 . The quantitative real-time polymerase chain reaction (qPCR) was conducted with the SYBR Premix ExTaq kit (TaKaRa) on the Applied Biosystems ViiA 7Dx Real-Time PCR system (Applied Biosystems, Shanghai, China). The standard curve for each target gene was established using gradient dilutions of the corresponding DNA fragments amplified by RT-PCR. Based on the standard curve, the mRNA copies of each target gene were determined. The qPCR was performed in duplicate for each sample. The expression levels of each target gene were shown as values normalized against human $10^{3}$ copies of GAPDH transcripts.

Table 1. Clinical Characteristics of Healthy Controls and Treatment-Naive Patients with Chronic Hepatitis B Virus Infection

\begin{tabular}{|c|c|c|c|c|c|}
\hline Group & $H C$ & $I T$ & $C H B$ & $I A$ & $\mathrm{p}$ \\
\hline$N$ & 28 & 15 & 31 & 20 & - \\
\hline Gender (male/female) & $15 / 13$ & $8 / 6$ & $17 / 14$ & $11 / 9$ & $\mathrm{NS}^{\mathrm{a}}$ \\
\hline Age (years) & $25(22-46)$ & $31(23-45)$ & $32(19-43)$ & $34(25-59)$ & $\mathrm{NS}^{\mathrm{b}}$ \\
\hline HBV DNA ( $\log _{10}$ copies/mL) & ND & $7.76(6.06-8.71)$ & $7.09(6.02-8.33)$ & $<3$ & - \\
\hline ALT (U/L) & $21(11-35)$ & $23(10-40)$ & $284(105-681)$ & $26(17-38)$ & $0.0001^{\mathrm{b}}$ \\
\hline $\mathrm{HBeAg} / \mathrm{anti}-\mathrm{HBe}$ & $0 / 0$ & $15 / 0$ & $31 / 0$ & $0 / 20$ & - \\
\hline
\end{tabular}

Values are $n$ or the median with range.

${ }^{a}$ Chi-squared test.

${ }^{\mathrm{b}}$ Kruskal-Wallis $\mathrm{H}$ test.

ALT, alanine aminotransferase; CHB, chronic hepatitis B; HBeAg, hepatitis B e antigen; HBV, hepatitis B virus; HC, healthy control; IA, inactive; IT, immune-tolerant; ND, not determined; NS, not significant. 
Table 2. Clinical Characteristics of Healthy Controls and Patients with Acute Hepatitis B Virus Infection and Varicella

\begin{tabular}{lcccc}
\hline Group & $H C$ & $A H B$ & $V Z V$ & $\mathrm{p}$ \\
\hline$n$ & 28 & 8 & 8 & - \\
Gender (male/female) & $15 / 13$ & $5 / 3$ & $4 / 4$ & $\mathrm{NS}^{\mathrm{a}}$ \\
Age (years) & $25(22-46)$ & $30(21-45)$ & $32(16-48)$ & $\mathrm{NS}^{\mathrm{b}}$ \\
HBV DNA (log $\log _{10}$ copies/mL) & $\mathrm{ND}$ & $3.44(2.10-5.03)$ & $\mathrm{ND}$ & $<0.0001^{\mathrm{b}}$ \\
ALT (U/L) & $21(11-35)$ & $517(100-1329)$ & $35(11-63)$ & $<0.0001^{\mathrm{b}}$ \\
TBil $(\mu \mathrm{mol} / \mathrm{L})$ & $12(6-16)$ & $67(35-125)$ & $11(5-21)$ & $<0.00$ \\
\hline
\end{tabular}

Values are $n$ or the median with range.

${ }^{\mathrm{a}}$ Chi-squared test.

${ }^{\mathrm{b}}$ Kruskal-Wallis $\mathrm{H}$ test.

AHB, acute hepatitis B; ALT, alanine aminotransferase; ND, not determined; TBil, total bilirubin; VZV, varicella zoster virus.

\section{Cell culture and stimulation}

PBMCs, monocytes, and nonmonocytes were cultured at $6 \times 10^{5}$ cells $/ \mathrm{mL}$ in RPMI 1640 containing $10 \%$ fetal bovine serum, $2 \mathrm{mmol} / \mathrm{L}$ L-glutamine, $100 \mu \mathrm{g} / \mathrm{mL}$ streptomycin, and $100 \mathrm{U} / \mathrm{mL}$ penicillin at $37^{\circ} \mathrm{C}$ in $5 \% \mathrm{CO}_{2}$ incubator. The fresh cells were cultured for $2 \mathrm{~h}$ and then stimulated with VACA ds 70 mer (InvivoGen) at a concentration of $2 \mu \mathrm{g} / \mathrm{mL}$ for $24 \mathrm{~h}$ according to the manufacturer's instructions. The mRNA expression of IFI16, STING, and IFN- $\beta$ was detected by qPCR.

\section{HBV DNA assays and serological assays}

Serum HBV DNA loads were quantified on the ABI 7500 Real-time PCR System (Applied Biosystems). The serum levels of hepatitis B surface antigen ( $\mathrm{HBsAg}), \mathrm{HBeAg}$, and anti-HBe were measured with electrochemiluminescence immunoassay on the Roche Diagnostics Cobas e601 analyzer (Roche Diagnostics, Germany). The normal range of serum ALT was 0-40 U/L.

\section{Statistical analysis}

Data were expressed as mean \pm standard deviation. GraphPad Prism 8 software was used for statistical analysis.
The Student's $t$-test was used for comparing the means of two groups. When comparing more than two groups, analyses were conducted using one-way analysis of variance, followed by the Bonferroni post hoc test. $p<0.05$ was considered significant.

\section{Results}

Upregulated mRNA expression of IFI16, DDX41, and MRE11 in PBMCs of chronic $H B V$-infected patients

We first assayed STING-dependent DNA sensors in PBMCs from chronic HBV-infected patients. Chronic HBVinfected patients were classified into three groups on the basis of their natural histories: IT carriers, characterized by HBeAg-positive with normal ALT levels and high HBV DNA loads $(>20,000 \mathrm{IU} / \mathrm{mL})$; immune-active patients, also known as "chronic hepatitis B" (CHB) patients, characterized by elevated ALT levels and viral loads $(>2,000 \mathrm{IU} / \mathrm{mL})$ with $\mathrm{HBeAg}$ or $\mathrm{HBeAb}$ positive; the inactive carriers, characterized by HBeAg-negative with normal ALT levels and low viral loads $(<2,000 \mathrm{IU} / \mathrm{mL})$. As shown in Figure $1 \mathrm{~A}$, IFI16 mRNA expression was upregulated in CHB patients. DDX41 and MRE11 mRNAs also increased in IT and IA patients as compared with those in HCs and CHB patients

Table 3. Primer Sequences Used for Quantitative Real-Time Polymerase Chain Reaction

\begin{tabular}{|c|c|c|c|c|}
\hline Gene name & GenBank accession & Primer type & Sequence $\left(5^{\prime} \rightarrow 3^{\prime}\right)$ & Amplicon size(bp) \\
\hline IFI16 & NM_005531.2 & Sense & GAAGTGCCAGCGTAACTCCTAA & 177 \\
\hline & & Antisense & TGATTGTGGTCAGTCGTCCAT & \\
\hline DDX41 & NM_016222.2 & Sense & TCCGTGAAAGAGCAGATGGAGA & 90 \\
\hline & & Antisense & СТTCTTCTGCAGCAAATCCATGAG & \\
\hline$D N A-P K$ & NM_006904.6 & $\begin{array}{l}\text { Sense } \\
\text { Anticense }\end{array}$ & GAAAGTTAGCAGGTGCCAATCCA & 101 \\
\hline$c G A S$ & NM_138441.2 & $\begin{array}{l}\text { Sense } \\
\text { Antisense }\end{array}$ & $\begin{array}{l}\text { GGGAGCTACTATGAGCACGTGAA } \\
\text { ACAAAGTAATATGCACGAGTGTTGG }\end{array}$ & 119 \\
\hline MRE11 & NM_005591.3 & $\begin{array}{l}\text { Sense } \\
\text { Antisense }\end{array}$ & $\begin{array}{l}\text { CTTGAGCATAAGGGTTATTTGAGCA } \\
\text { CAAGTTCAATAAAGATGTGGGCAGA }\end{array}$ & 102 \\
\hline$D A I$ & NM_030776.2 & $\begin{array}{l}\text { Sense } \\
\text { Antisense }\end{array}$ & $\begin{array}{l}\text { TGGGGAGGTGGGATTTAGTG } \\
\text { TCTGGAAGCAAGCAGGTCAA }\end{array}$ & 131 \\
\hline STING & NM_001301738 & $\begin{array}{l}\text { Sense } \\
\text { Antisense }\end{array}$ & $\begin{array}{l}\text { САСССТTСТССССТТССТТT } \\
\text { ССТССТССТССТСТССАТТСТТ }\end{array}$ & 133 \\
\hline$I F N-\beta$ & NM_002176 & $\begin{array}{l}\text { Sense } \\
\text { Antisense }\end{array}$ & $\begin{array}{l}\text { ACAGGTTACCTCCGAAACTGAAGA } \\
\text { TTAGCCATCAGTCACTTAAACAGCA }\end{array}$ & 108 \\
\hline$G A P D H$ & NM_002046.5 & $\begin{array}{l}\text { Sense } \\
\text { Antisense }\end{array}$ & $\begin{array}{l}\text { GCACCGTCAAGGCTGAGAAC } \\
\text { TGGTGAAGACGCCAGTGGA }\end{array}$ & 138 \\
\hline
\end{tabular}




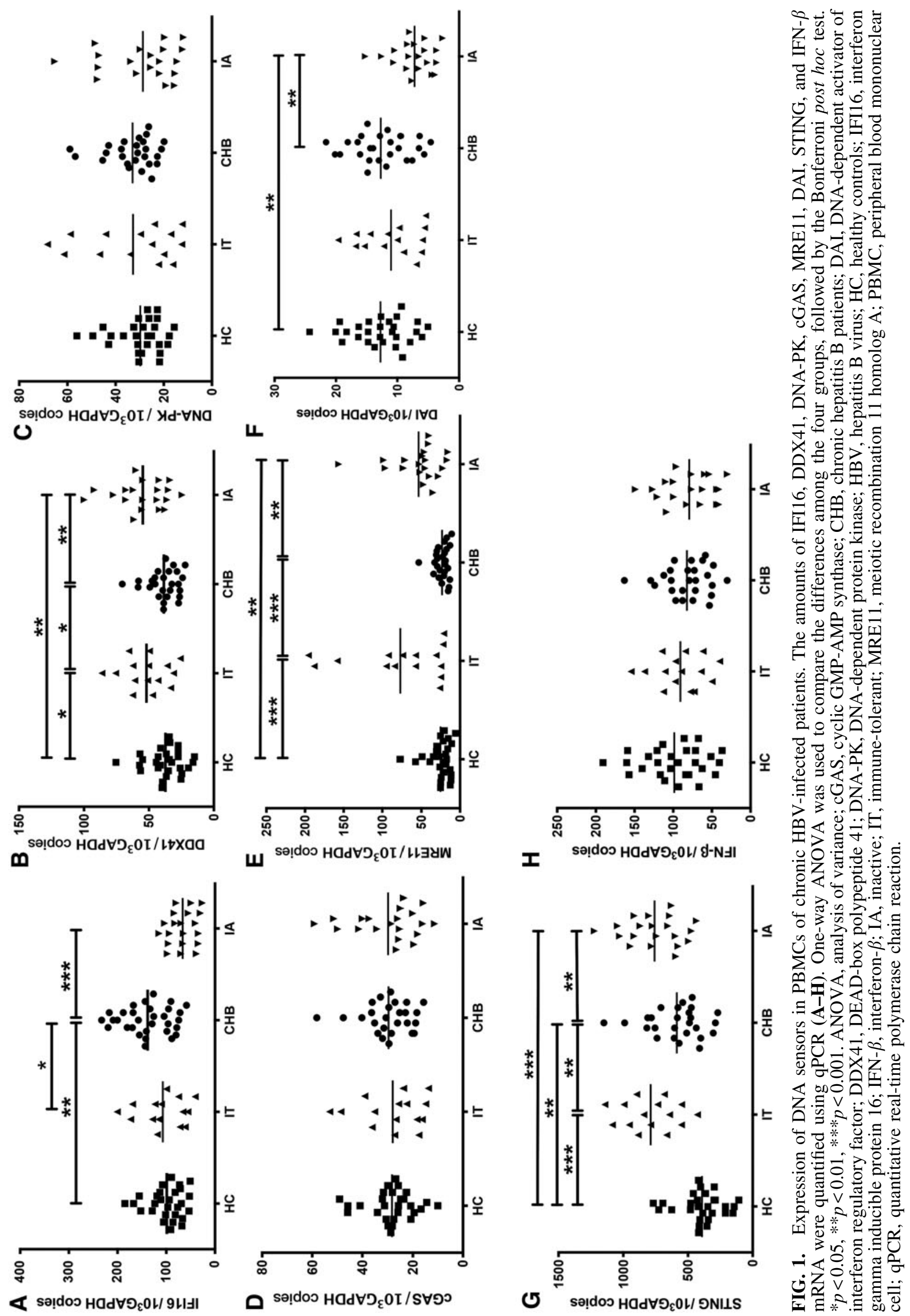


(Fig. 1B, E). Despite the anti-HBV effect of cGAS and DAI in cell culture and animal models $(5,7,15)$, the cGAS and DAI expression was not enhanced in the chronic HBV-infected groups (Fig. 1D, F), and DNA-PK expression did not change as well (Fig. 1C). In addition, as shown in Supplementary Figure S1, the expression levels of IFI16 and DDX41 mRNA were significantly positively correlated with the $\mathrm{CHB}$ patients' HBV DNA loads $(r=0.6578, p<0.0001 ; r=0.5889$, $p=0.0016$ ) (Supplementary Fig. S1A, B). Enhanced serum IFI16 protein levels were also observed in $\mathrm{CHB}$ patients (Supplementary Fig. S2). With enhanced expression of IFI16, DDX41, and MRE11 in CHB, IT, and IA patients, the adaptor STING expression was also upregulated in all chronic HBVinfected groups (Fig. 1G). Interestingly, we did not observe the induction of the downstream effector cytokines IFN- $\beta$ (Fig. $1 \mathrm{H})$ and TNF- $\alpha$ (data not shown), in keeping with other evidence showing that HBV polymerase could interfere with STING ubiquitination modification to suppress IRF3 activation and IFN- $\beta$ expression (26). Taken together, these data suggest that HBV might be sensed by intracellular DNA sensors in PBMCs during chronic HBV infection but may exert opposite effects on activation of the DNA sensorSTING pathway.

\section{IFI16 expression is upregulated in PBMCs of $A H B$ patients}

The incidence of $\mathrm{AHB}$ is extremely low $(<4.4$ per 100,000 population) $(11,17,29,34)$, making it difficult to conduct clinical studies. Therefore, AHB is always less understood as compared with chronic HBV infection and the contribution of innate immunity to the control of AHB is even less reported. Varicella is an acute and self-limited infectious disease caused by VZV. VZV and HBV are both DNA viruses. However, in contrast to AHB, VZV infection of healthy people at any age is almost always self-limited. Therefore, we used patients with varicella as the control cases of AHB to compare differences in the expression of DNA sensors in PBMCs.

As shown in Figure 2A, only IFI16 expression was upregulated in $\mathrm{AHB}$ patients as compared with $\mathrm{HCs}$, and other DNA sensors in AHB patients were strikingly downregulated (Fig. 2B-F), analogous to the reduced innate immune responses in AHB patients in a previous study (12). More interesting is, except IFI16, all the other DNA sensors in varicella were also significantly downregulated (Fig. 2B-F). Despite the elevated expression of IFI16, IFN- $\beta$ expression was not altered in AHB (Fig. 2H), supporting the idea of a lack of induction of IFN-Is in AHB (12). These results suggest that HBV might be sensed by IFI16 in acute HBV infection and can counteract the induction of IFN- $\beta$ in PBMCs.

\section{IFI16 is mainly expressed in monocytes of $A H B$ and $C H B$ patients}

As enhanced IFI16 expression was involved in both CHB and AHB, we next investigated the cellular source of IFI16. Previous studies suggested that IFI16 is mainly expressed in the $\mathrm{CD} 14^{+}$monocyte lineage $(2,8)$. Besides the monocyte lineage, lymphoid cell lines also constitutively express IFI16, but the expression is not affected by IFNs $(8,9)$. Therefore, to compare IFI16 expression in immune cells, we divided PBMCs into monocytes and nonmonocytes. As shown in Figure 3, IFI16 was mainly expressed in monocytes in HCs $(p<0.001)$ and its expression level was significantly upregulated in monocytes from $\mathrm{AHB}$ and $\mathrm{CHB}$ patients, suggesting that HBV might be sensed by monocytes, which is in keeping with previous studies (6). In addition, IFI16 expression levels in PBMCs were significantly positively correlated with serum HBV DNA loads in CHB patients (Supplementary Fig. S1A). Taken together, these findings imply that HBV might be sensed by monocytes and its infection induces enhanced expression of IFI16 in monocytes of $\mathrm{AHB}$ and $\mathrm{CHB}$ patients.

\section{Stimulation of IFI16 expression by its ligand VACV ds $70 m e r$ is attenuated in monocytes from $\mathrm{CHB}$ patients}

Although HBV infection resulted in enhanced expression of IFI16 and STING in CHB patients, we did not observe increased expression of IFN- $\beta$ in $\mathrm{CHB}$ patients accordingly. We speculate that the IFI16-STING-IFN- $\beta$ pathway may be suppressed by HBV in monocytes of $\mathrm{CHB}$ patients. Previous studies showed that as a ligand for IFI16, the VACV doublestranded DNA 70-base pairs motif (VACV ds 70mer) can potently induce IFN- $\beta$ in PBMCs and monocytes (38). To confirm our speculation, PBMCs and monocytes were isolated from $\mathrm{HCs}$ and $\mathrm{CHB}$ patients and stimulated in vitro by VACV ds 70mer to assay the response of the IFI16-STINGIFN- $\beta$ pathway. As shown in Figure $4 \mathrm{~A}-\mathrm{D}$, the expression of IFI16 and STING in PBMCs and monocytes of CHB patients was significantly attenuated as compared with $\mathrm{HCs}$ after $24 \mathrm{~h}$ of stimulation by VACV ds 70 mer. As a result, the IFN- $\beta$ response to $\mathrm{VACV}$ ds 70 mer was severely suppressed in PBMCs and monocytes of CHB patients (Fig. 4E, F). These results support our speculation that the response of the IFI16-STING-IFN- $\beta$ pathway to $\mathrm{HBV}$ is attenuated in monocytes of $\mathrm{CHB}$ patients, consistent with the finding that $\mathrm{HBV}$ is recognized mainly by liver macrophages (Kupffer cells) but does not induce an interferon response (16).

\section{Discussion}

Capable of recognition of pathogenic DNA, the innate immune defense system composed of intracellular DNA sensors plays an important role in preventing intracellular infection of pathogens such as DNA viruses. However, their role in HBV infection is still largely unclear. Published studies have shown that early in acute infection, HBV is poorly sensed by innate immunity and does not or weakly induce innate immune responses $(12,31,41,42)$. It incubates stealthily in the liver for several weeks until elicitation of adaptive immune responses. Little is known about the involvement of DNA sensors in the process of acute and chronic HBV infection in humans. Collectively, our results reveal that (1) HBV might be sensed by DNA sensors in acute and chronic HBV-infected patients, (2) IFI16 expression is enhanced in PBMCs of both $\mathrm{AHB}$ and $\mathrm{CHB}$ patients and it is mainly expressed in monocytes, and (3) the IFI16-STING pathway in monocytes is attenuated by HBV infection.

The intracellular recognition of $\mathrm{HBV}$ infection by DNA sensors is still less understood. Moreover, it remains controversial in whether HBV can be sensed by intracellular DNA sensors or actively evades from the sensing pathways. To address this issue, we first analyzed the expression of 


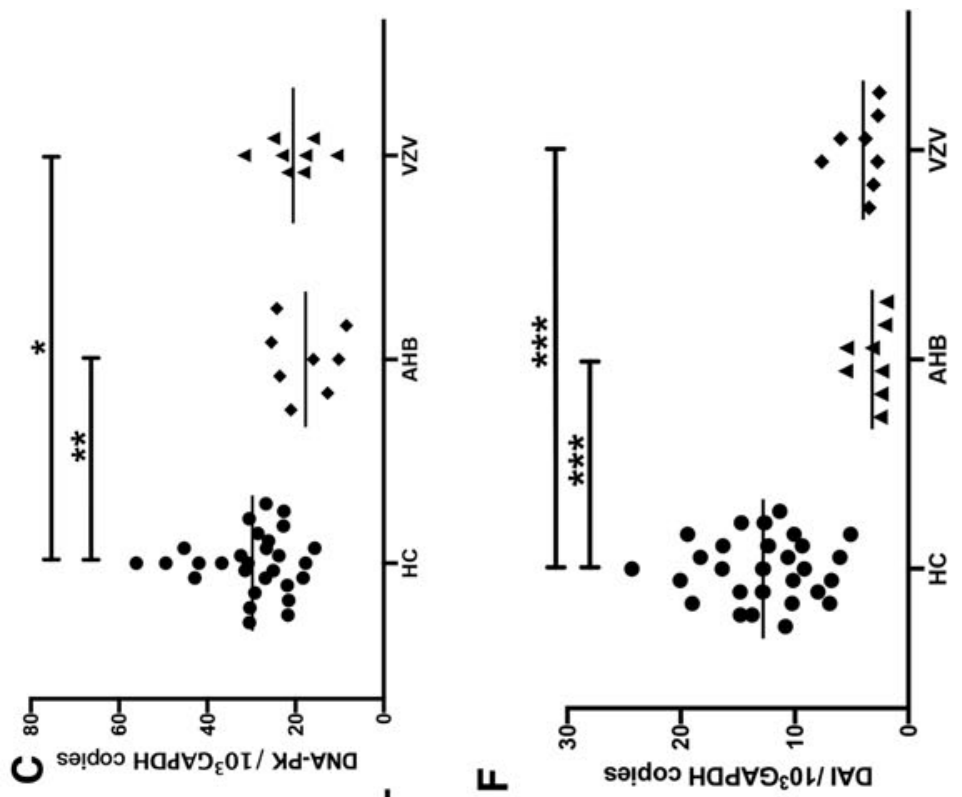

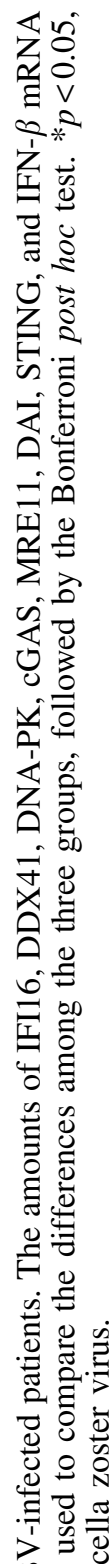

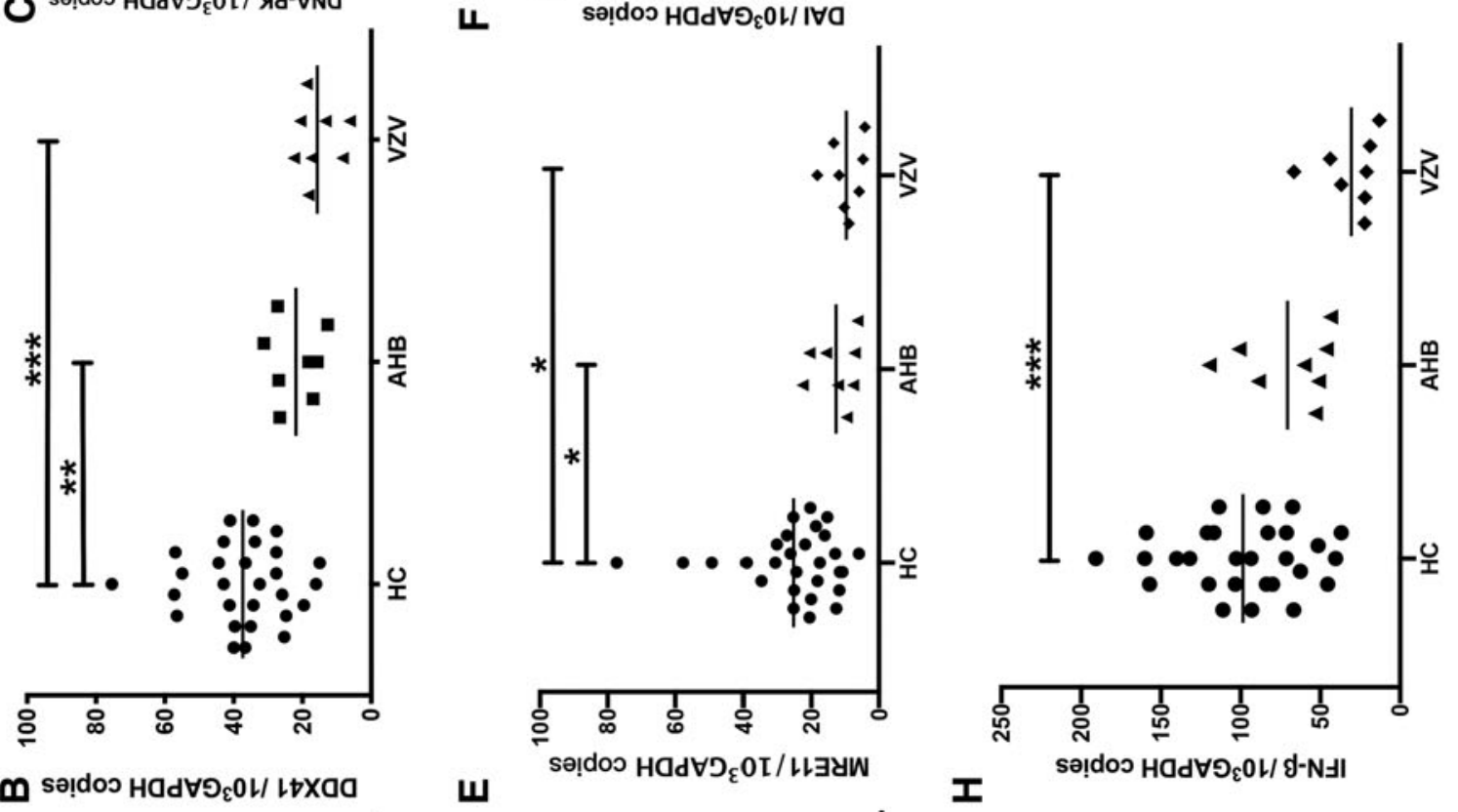

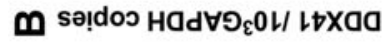

ш

I

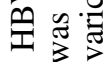

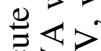

שㅇㄱ

OZ

is $<\infty$

完:

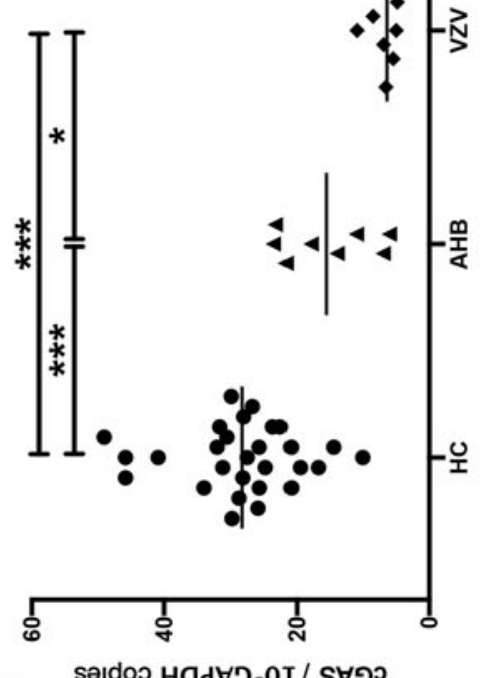

$\cdot \cdot \cdot \div \quad$ -

年

$\Xi 0$

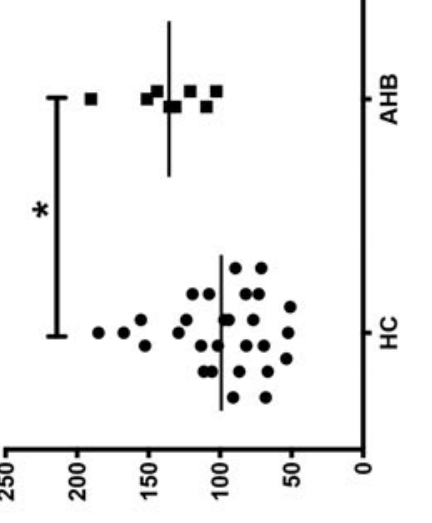

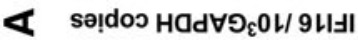



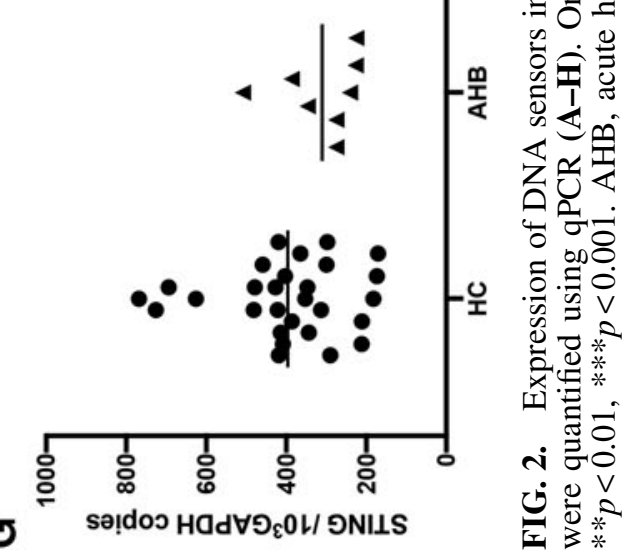




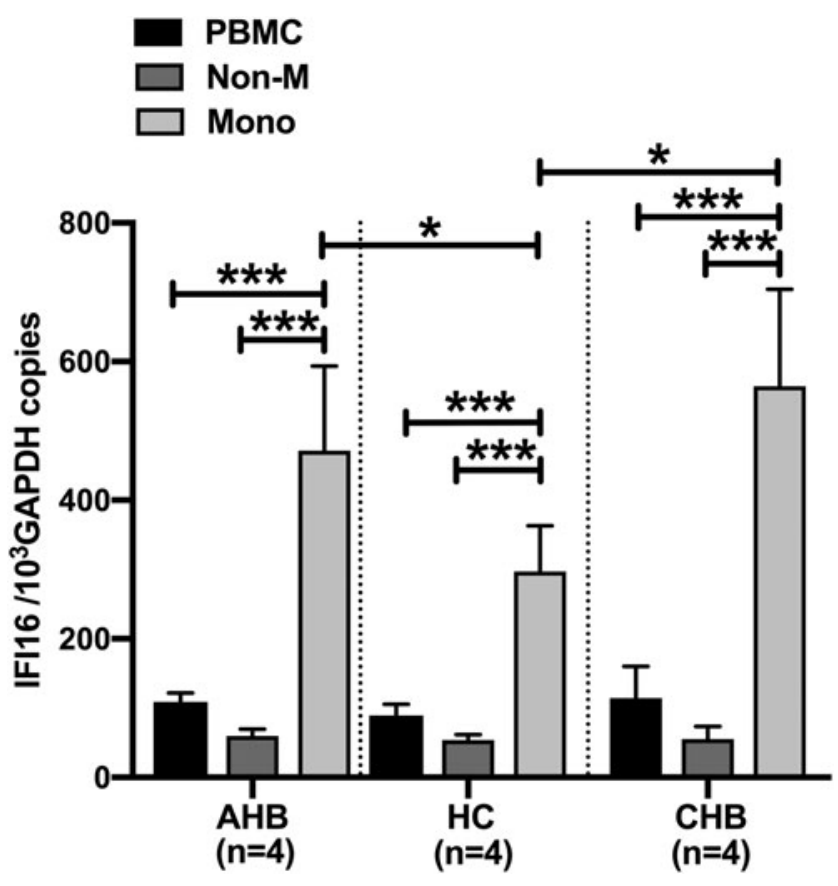

FIG. 3. IFI16 expression in PBMCs, monocytes, and nonmonocytes. IFI16 mRNA expression was determined by qPCR. Statistical analyses were performed using a Student's $t$-test. $* p<0.05, * * * p<0.001$.

STING-dependent DNA sensors in PBMCs of chronic HBV-infected patients. The results showed the expression of IFI16, DDX41, and MRE11 was enhanced in CHB, IT, and IA patients. Published data showed that IFI16 was closely related to the degree of liver inflammation in $\mathrm{CHB}$ patients (28). Upregulated expression of IFI16 only in chronic hepatitis patients suggests that it may be associated with inflammation within the liver. It was recently demonstrated that IFI16 could recognize and directly bind to HBV covalently closed circular DNA (cccDNA) through an interferonstimulated response element (ISRE) within the cccDNA in hepatocytic nuclei (43). If this is the case, the enhanced expression of IFI16 in PBMCs occurs only when cccDNA is exposed in blood through inflammatory necrosis of hepatocytes, as HBV cccDNA only originates from hepatocytes, not from PBMCs (19). This may explain why IFI16 expression in PBMCs is upregulated only in acute or chronic hepatitis patients, but not in IT or IA groups that have normal ALT levels. DDX41 and MRE11 were reported to serve as cytosolic dsDNA sensors by Liu's and Kawai's groups, respectively $(20,45)$. The DDX41 and MRE11 expression increased both in IT and IA groups, suggesting that they were involved in chronic HBV infection. Although there is still no report concerning the role of DDX41 and MRE11 playing in HBV infection, other reports have shown that they are involved in the control of virus infections $(14,24,32)$. Thus, further investigation of their roles in HBV infection is warranted. Although activation of the cGASSTING pathway was demonstrated to be able to inhibit HBV replication in cell culture and mice $(7,15,18,40)$, HBV could also actively suppress cGAS expression and function in vitro and humanized liver chimeric mice (40). Our results showed cGAS expression level was not altered in chronic
HBV patients, suggesting HBV may evade from cGAS sensing. In parallel with the increased expression of IFI16, DDX41, and MRE11, the adaptor STING expression was also enhanced in chronic HBV patients. However, the induction of the effector IFN- $\beta$ was not observed in chronic HBV patients, implying the DNA sensor-STING pathway is suppressed. This may be explained by Liu's report showing HBV polymerase blocked DNA-sensing pathways by disrupting k63-linked ubiquitination of STING (26). Taken together, these data suggest HBV might be sensed by DNA sensors in PBMCs in chronic HBV-infected patients and support the notion that HBV plays an active role in evading and inhibiting innate immune responses (13).

Different from the expression profile of DNA sensors in chronic HBV infection, IFI16 was found to be the only upregulated DNA sensor in AHB patients, in accordance with a previous report that showed induced expression of the IFI16 gene in an acute HBV infection model of chimpanzees (41). However, the other DNA sensors were significantly downregulated as compared with HCs. This is reminiscent of the report by Dunn in which he demonstrated that with abundant production of IL-10 in AHB patients, the responses of $\mathrm{NK}$ cells along with $\mathrm{CD}^{+}$and $\mathrm{CD}^{+} \mathrm{HBV}-$ specific $\mathrm{T}$ cells from PBMCs were reduced (12). Interestingly, varicella patients and AHB patients displayed a similar expression pattern of DNA sensors, except IFI16. Despite with severe liver injury and a rapid short-term drop in HBV DNA levels in these AHB patients, the STING and IFN- $\beta$ expression was not induced by acute HBV infection. The absence of a robust IFN-I response in AHB is in accordance with other studies $(12,41)$. Of note, IFN- $\beta$ expression in varicella patients was significantly suppressed as compared with HCs, which may be explained by the evidence that IFN- $\beta$ expression is inhibited by VZV ORF47 kinase through preventing IRF3 activation in VZV-infected cells (39). Taken together, these data suggest that HBV might be sensed by IFI16 in acute HBV infection and utilize an immunosuppressive strategy to actively prevent innate immune responses.

Next, we determined the cellular source of IFI16. Early reports showed IFI16 was constitutively expressed in all $\mathrm{CD} 4^{+}$myeloid progenitor cells from human bone marrow and later exclusively in the $\mathrm{CD} 14^{+}$monocytoid lineage (8). IFI16 is present in peripheral blood monocytes and lymphocytes, but not in granulocytes. In lymphocytes, it is not regulated by IFN- $\alpha$, IFN- $\gamma$, and IL6 (9). In 2010, IFI16 was first described as a STING-dependent dsDNA sensor in monocytes and macrophages by Bowie's group (38). Recently, Liang's group revealed that HBV was not sensed by the infected hepatocytes due to the lack of DNA sensing machinery but could induce human peripheral macrophage activation (6). Based on these data, we divided PBMCs into monocytes and nonmonocytes by magnetic separation. Quantitative RT-PCR analysis revealed that IFI16, whether in $\mathrm{HCs}$, the $\mathrm{CHB}$ group, or the AHB group, is mainly expressed in monocytes. Furthermore, IFI16 expression in monocytes from both AHB and CHB patients was significantly upregulated as compared with that from HCs. These findings, along with the data from the aforementioned studies, indicate that HBV infection results in enhanced expression of IFI16 in monocytes of $\mathrm{AHB}$ and $\mathrm{CHB}$ patients. 



FIG. 4. Stimulation of IFI16 expression by VACV ds 70mer is attenuated in PBMCs and monocytes from CHB patients. PBMCs and monocytes from CHB patients were MT or transfected for 6 or $24 \mathrm{~h}$ with $2 \mu \mathrm{g} / \mathrm{mL}$ of VACV ds $70 \mathrm{mer}$. The transcripts of IFI16, STING, and IFN- $\beta$ were then measured by qPCR (A-F). Data depicted represent mean \pm SD of five independent patients from one of two experiments showing similar results. Statistical analyses were performed using a Student's $t$-test. $* p<0.05, * * p<0.01, * * * p<0.001$. CHB, chronic hepatitis B patients.

Finally, we investigated the response of the IFI16-STING pathway in monocytes to VACV ds 70mer, a ligand for IFI16, to further confirm the impact of HBV infection on IFN- $\beta$ expression. In concordance with the results from patients, the response of the IFI16-STING pathway to VACV ds 70mer was significantly attenuated in monocytes of $\mathrm{CHB}$ patients in comparison with $\mathrm{HCs}$, suggesting that this DNA sensing pathway is impaired in CHB patients. These data further support the view that HBV is not always invisible to innate immunity but has evolved strategies to actively counteract recognition by innate immunity (13).
Our study has some limitations. First, the expression of DNA sensors in PBMCs may not be representative of the expression profile in liver. Therefore, studies of liver tissues should be included. Second, more cases of AHB patients are needed to verify the expression characteristics of these DNA sensors in AHB. Third, the response of the IFI16-STING pathway to VACV ds 70mer in monocytes from AHB patients was not assayed because of the difficulty of obtaining adequate specimens from AHB patients. Thus, additional studies are warranted. Finally, the detailed mechanism of suppression of the IFI16-STING pathway by HBV is needed to identify. 
In summary, we investigated for the first time the role of STING-dependent DNA sensors in humans with acute and chronic HBV infection. Our data suggest that HBV might be sensed by DNA sensors in PBMCs of acute and chronic $\mathrm{HBV}$-infected patients, and meanwhile HBV infection also attenuates the response of the DNA sensor-STING pathway in PBMCs and monocytes from CHB patients, which may facilitate the persistence of $\mathrm{HBV}$ infection. These findings increase our understanding of HBV immune pathogenesis and warrant further investigations to explore the profound mechanisms by which HBV suppresses the DNA sensing pathway.

\section{Acknowledgments}

We thank all the participants in this study. We also thank Dr. Yaying Zhou for flow cytometric analysis.

\section{Author Disclosure Statement}

No competing financial interests exist.

\section{Funding Information}

This study was supported by the Committee of Innovation of Science and Technology of Shenzhen (No. JCYJ20150403101028209).

\section{Supplementary Material}

Supplementary Figure S1

Supplementary Figure S2

\section{References}

1. Aparna S, Johannes H, Mikolajczyk RT, et al. Estimations of worldwide prevalence of chronic hepatitis B virus infection: a systematic review of data published between 1965 and 2013. Lancet 2015;386:1546-1555.

2. Bourette RP, and Mouchiroud G. The biological role of interferon-inducible P204 protein in the development of the mononuclear phagocyte system. Front Biosci 2008;13: 879-886.

3. Cai X, Chiu YH, and Chen ZJ. The cGAS-cGAMP-STING pathway of cytosolic DNA sensing and signaling. Mol Cell 2014;54:289-296.

4. Chen H, He G, Chen Y, et al. Differential activation of NLRP3, AIM2, and IFI16 inflammasomes in humans with acute and chronic hepatitis B. Viral Immunol 2018;31:639-645.

5. Chen QY, Liu YH, Li JH, et al. DNA-dependent activator of interferon-regulatory factors inhibits hepatitis B virus replication. World J Gastroenterol 2012;18:2850-2858.

6. Cheng X, Xia Y, Serti E, et al. Hepatitis B virus evades innate immunity of hepatocytes but activates cytokine production by macrophages. Hepatology 2017;66:17791793.

7. Dansako H, Ueda Y, Okumura N, et al. The cyclic GMPAMP synthetase-STING signaling pathway is required for both the innate immune response against HBV and the suppression of HBV assembly. FEBS J 2016;283:144-156.

8. Dawson MJ, Elwood NJ, Johnstone RW, et al. The IFNinducible nucleoprotein IFI 16 is expressed in cells of the monocyte lineage, but is rapidly and markedly downregulated in other myeloid precursor populations. J Leukoc Biol 1998;64:546-554.
9. Dawson MJ, and Trapani JA. IFI 16 gene encodes a nuclear protein whose expression is induced by interferons in human myeloid leukaemia cell lines. J Cell Biochem 1995;57:39-51.

10. Dempsey A, and Bowie AG. Innate immune recognition of DNA: a recent history. Virology 2015;479-480:146-152.

11. Duffell EF, van de Laar MJ, and Amato-Gauci AJ. Enhanced surveillance of hepatitis B in the EU, 2006-2012. J Viral Hepat 2015;22:581-589.

12. Dunn C, Peppa D, Khanna P, et al. Temporal analysis of early immune responses in patients with acute hepatitis B virus infection. Gastroenterology 2009;137:1289-1300.

13. Ferrari C. HBV and the immune response. Liver Int 2015; 35 Suppl 1:121-128.

14. Fullam A, and Schroder M. DExD/H-box RNA helicases as mediators of anti-viral innate immunity and essential host factors for viral replication. Biochim Biophys Acta 2013; 1829:854-865.

15. He J, Hao R, Liu D, et al. Inhibition of hepatitis B virus replication by activation of the cGAS-STING pathway. J Gen Virol 2016;97:3368-3378.

16. Hosel M, Quasdorff M, Wiegmann K, et al. Not interferon, but interleukin-6 controls early gene expression in hepatitis B virus infection. Hepatology 2009;50:1773-1782.

17. Hyun Kim B, and Ray Kim W. Epidemiology of hepatitis B virus infection in the United States. Clin Liver Dis (Hoboken) 2018;12:1-4.

18. Imai H, Dansako H, Ueda Y, et al. Daunorubicin, a topoisomerase II poison, suppresses viral production of hepatitis B virus by inducing cGAS-dependent innate immune response. Biochem Biophys Res Commun 2018;504: $672-678$

19. Kock J, Theilmann L, Galle P, et al. Hepatitis B virus nucleic acids associated with human peripheral blood mononuclear cells do not originate from replicating virus. Hepatology 1996;23:405-413.

20. Kondo T, Kobayashi J, Saitoh T, et al. DNA damage sensor MRE11 recognizes cytosolic double-stranded DNA and induces type I interferon by regulating STING trafficking. Proc Natl Acad Sci U S A 2013;110:2969-2974.

21. Lai CL, Ratziu V, Yuen MF, et al. Viral hepatitis B. Lancet 2003;362:2089-2094.

22. Li Y, Wilson HL, and Kiss-Toth E. Regulating STING in health and disease. J Inflamm (Lond) 2017;14:11.

23. Liu $H$, and Zhang $X$. Innate immune recognition of hepatitis B virus. World J Hepatol 2015;7:2319-2322.

24. Liu J, Huang Y, Huang X, et al. Grouper DDX41 exerts antiviral activity against fish iridovirus and nodavirus infection. Fish Shellfish Immunol 2019;91:40-49.

25. Liu S, Cai X, Wu J, et al. Phosphorylation of innate immune adaptor proteins MAVS, STING, and TRIF induces IRF3 activation. Science 2015;347:aaa2630.

26. Liu Y, Li J, Chen J, et al. Hepatitis B virus polymerase disrupts K63-linked ubiquitination of STING to block innate cytosolic DNA-sensing pathways. J Virol 2015;89: 2287-2300.

27. Paludan SR, and Bowie AG. Immune sensing of DNA. Immunity 2013;38:870-880.

28. Pang X, Li X, Mo Z, et al. IFI16 is involved in HBVassociated acute-on-chronic liver failure inflammation. BMC Gastroenterol 2018;18:61.

29. Sagnelli E, Sagnelli C, Pisaturo M, et al. Epidemiology of acute and chronic hepatitis B and delta over the last 5 decades in Italy. World J Gastroenterol 2014;20:76357643 . 
30. Scutts SR, Ember SW, Ren H, et al. DNA-PK is targeted by multiple vaccinia virus proteins to inhibit DNA sensing. Cell Rep 2018;25:1953-1965 e1954.

31. Stacey AR, Norris PJ, Qin L, et al. Induction of a striking systemic cytokine cascade prior to peak viremia in acute human immunodeficiency virus type 1 infection, in contrast to more modest and delayed responses in acute hepatitis B and C virus infections. J Virol 2009;83:3719-3733.

32. Stavrou S, Aguilera AN, Blouch K, et al. DDX41 recognizes RNA/DNA retroviral reverse transcripts and is critical for in vivo control of murine leukemia virus infection. MBio 2018;9:e00923-18.

33. Stempel M, Chan B, and Brinkmann MM. Coevolution pays off: herpesviruses have the license to escape the DNA sensing pathway. Med Microbiol Immunol 2019;208:495512.

34. Su WJ, Liu CC, Liu DP, et al. Effect of age on the incidence of acute hepatitis B after 25 years of a universal newborn hepatitis B immunization program in Taiwan. J Infect Dis 2012;205:757-762.

35. Suslov A, Boldanova T, Wang X, et al. Hepatitis B virus does not interfere with innate immune responses in the human liver. Gastroenterology 2018;154:1778-1790.

36. Terrault NA, Bzowej NH, Chang KM, et al. AASLD guidelines for treatment of chronic hepatitis B. Hepatology 2016;63:261-283.

37. Unterholzner L, and Almine JF. Camouflage and interception: how pathogens evade detection by intracellular nucleic acid sensors. Immunology 2019;156:217-227.

38. Unterholzner L, Keating SE, Baran M, et al. IFI16 is an innate immune sensor for intracellular DNA. Nat Immunol 2010;11:997-1004.

39. Vandevenne $\mathrm{P}$, Lebrun $\mathrm{M}$, El Mjiyad $\mathrm{N}$, et al. The varicella-zoster virus ORF47 kinase interferes with host innate immune response by inhibiting the activation of IRF3. PLoS One 2011;6:e16870.
40. Verrier ER, Yim SA, Heydmann L, et al. Hepatitis B virus evasion from cyclic guanosine monophosphate-adenosine monophosphate synthase sensing in human hepatocytes. Hepatology 2018;68:1695-1709.

41. Wieland S, Thimme R, Purcell RH, et al. Genomic analysis of the host response to hepatitis B virus infection. Proc Natl Acad Sci U S A 2004;101:6669-6674.

42. Wieland SF, and Chisari FV. Stealth and cunning: hepatitis B and hepatitis C viruses. J Virol 2005;79:9369-9380.

43. Yang Y, Zhao X, Wang Z, et al. Nuclear sensor interferoninducible protein 16 inhibits the function of hepatitis $B$ virus covalently closed circular DNA by integrating innate immune activation and epigenetic suppression. Hepatology 2020;71:1154-1169.

44. Zhang C, Shang G, Gui X, et al. Structural basis of STING binding with and phosphorylation by TBK1. Nature 2019; 567:394-398.

45. Zhang Z, Yuan B, Bao M, et al. The helicase DDX41 senses intracellular DNA mediated by the adaptor STING in dendritic cells. Nat Immunol 2011;12:959-965.

Address correspondence to: Prof. Xiaoyong Zhang State Key Laboratory of Organ Failure Research Guangdong Provincial Key Laboratory of Viral Hepatitis Research

Department of Infectious Diseases Nanfang Hospital

Southern Medical University No. 1838, North Guangzhou Avenue Guangzhou 510515

China

E-mail: xiaoyzhang@smu.edu.cn 